\title{
Educación secundaria y deporte de alta dedicación: Estudio cualitativo de modelos de compaginación Secondary education and high dedication sport: Qualitative study of the compatibility programs Josep Solà Santesmases \\ Universitat Ramon Llull (España)
}

Resumen. El artículo tiene por objetivo comprender los modelos educativos para compaginar los estudios y el deporte de alta dedicación en la educación secundaria, y la implicación de los clubs en su gestión. Se analizan el modelo no integrado con proyecto para deportistas (adaptación curricular sin responsabilidad en el deporte) y el modelo integrado (currículum y deporte bajo su responsabilidad). Conscientes que son pocos los deportistas beneficiados con ayudas institucionalizadas, se plantea la eficacia de dichos modelos de compaginación para atender al resto de estudiantes deportistas. Desde un enfoque cualitativo y descriptivo, se entrevistaron a directores de centros públicos y privados, además de la inspección educativa (n=5), y también a directores técnicos de un deporte de equipo (baloncesto) y de un deporte individual (natación), con exigencias diferenciadas (n=9). Para ello se elaboró una entrevista semi-estructurada dividida en tres bloques: génesis y estructura del proyecto; medidas concretas de compaginación de estudios y deporte, y prospectiva de futuro. Los resultados muestran una mayor sensibilidad docente y jurídica hacia los estudiantes deportistas, aunque los modelos educativos que los apoyan están aún en fase de desarrollo. La variabilidad de los indicadores estudiados dificulta la cohesión de soluciones para una armonización efectiva del deporte y la escuela. Esto provoca que se mantengan problemas endémicos todavía no resueltos a nivel político: profundización legal de proyectos, adaptación similar a los estudios de música o danza, y flexibilización racional del calendario.

Palabras clave. Educación secundaria, Deporte de alta dedicación, Modelos de Compaginación, Proyectos de centro para deportistas, Implicación del club en los estudios.

Abstract. The aim of this article is to understand the educational models, which seek to combine studies and high dedication sport in secondary education, and the involvement of clubs in their management. We analyzed the model with a non-integrated project for athletes (adapted curriculum without responsibility to the sport), and the integrated model (studies and sport under its responsibility). Given the awareness that few athletes benefit from institutionalized aids, the effectiveness of these models of compatibility is examined in order to help other student athletes. In addition to the educational inspection, we interviewed directors of public and private schools $(n=5)$ as well as technical directors of a team sport (basketball) and an individual sport (swimming), with different requirements $(\mathrm{n}=9$ ), from a qualitative and descriptive approach. In order for this to be done, a semistructured interview divided into three blocks was developed: genesis and structure of the project; concrete measures to combine studies and sport, and future prospects. The results showed a greater legal and educational sensitivity toward student-athletes, although the educational models that support them are still in a developmental phase. The variability of the studied indicators hinders the cohesion of solutions for an effective harmonization between school and sport. Due to this, endemic problems such as the legal deepening of projects, similar adaptation as in the studies of music or dance and rational flexibility of the calendar, remain unsolved at the political level.

Keywords. Secondary education, High dedication sport, Models of Compatibility, School projects for athletes, Club involvement in studies.

\section{Introducción}

Desde la psicopedagogía del deporte, un tema de reflexión recurrente estudia si la dedicación exigente a los entrenamientos puede afectar al rendimiento académico de los estudiantes deportistas. Preocupación de muchas familias que enfatiza la necesidad del compromiso político en la investigación de la efectividad de los modelos educativos para compaginar estudio y deporte (Surís \& Parera, 2005; Cano, 2008; Solà, 2010). Existen diferentes revisiones internacionales sobre la temática que no aportan resultados concluyentes (Taras, 2005; Sing, Uijtdewilligen, Twisk, Van Mechelen \& Chinapaw, 2012). Los modelos de compaginación que se presentan dependen de los contextos sociales donde se aplican, impidiendo su generalización. Por este motivo, se aportaran referencias sobre la relación entre la formación académica y el deporte de alta dedicación, además de las principales medidas jurídicas específicas de nuestro país.

Heinemann (1998) afirma que a medida que los deportistas dedican más tiempo a entrenar, menores son las posibilidades de prepararse para el futuro. De esta manera, el deporte de alta dedicación dificulta la compaginación con la formación académica, puesto que ambas actividades coinciden en la adolescencia y plantean una crisis entre lo que se desea y la imposibilidad de realizarlo (Stambulova, 2000). Al agotarse la vida deportiva, si la formación académica resulta inferior respecto al resto de la población, la profesionalización futura queda en entredicho (Vilanova, 2009; Vilanova \& Puig, 2013). En este sentido, Ceciæ Erpiè, Wylleman, \& Zupancic (2004) afirman que el nivel educativo tiene relación con futuras dificultades económicas y de inserción laboral. Disponer de una buena formación académica aumenta los conocimientos profesionales y las oportunidades laborales (Murphy, Petitpas \& Brewer, 1996; Williams-Ryce, 1996; Ceciæ Erpiè, et. al., 2004). Son

Fecha recepción: 14-10-14- Fecha envío revisores: 24-10-14- Fecha de aceptación: 17-03-15 Josep Solà Santesmases josepss@blanquerna.url.edu muchos los estudios que demuestran que al finalizar la carrera deportiva, disponer de formación académica influye positivamente en la calidad de la retirada (Werthner \& Orlick, 1986; Wylleman, De Knop, Menkehorst, Theeboom, \&Annerel, 1993; González \& Bedoya, 2008).

Desde el ámbito institucional, el Comité Olímpico Internacional (COI), ha diseñado el Athlete Career Programm para promover el estudio en los deportistas de élite. En la misma línea ha actuado la Comisión Europea, con el proyecto Dual Career. Vilanova (2009) y Regüela (2011) destacan que en Estados Unidos la universidad es garantía de continuidad en la excelencia deportiva. En Europa, los institutos de secundaria se ubican en los centros de alto rendimiento, donde una minoría de deportistas estudia en un modelo integrado. Wylleman \& Lavallee (2004) destacan las topsport schools en Bélgica (Flandes), centros de secundaria ordinarios que adaptan el currículum con veinte horas semanales de educación general obligatoria, junto con doce horas de entrenamiento deportivo específico (que incluyen la educación física). Los Juegos Olímpicos de Barcelona 1992 y la reforma educativa permeabilizaron la legislación ayudando a los jóvenes deportistas (Cano, 2008). Anivel universitario, Torregrosa, Sánchez \& Cruz (2004) destacan los programas de compaginación de la carrera deportiva con el desarrollo académico vocacional en la Universidad Autónoma de Barcelona, pionera en su relación con el Centro de Alto Rendimiento (CAR). El programa Tutorsport, estructurado en tutorías individualizadas, se ha generalizado al resto de universidades como ítem de calidad.

Pocos ciudadanos realizan dos actividades tan exigentes como los deportistas de alto nivel, planteándose la posibilidad que éstos enfoquen su vida de manera diferencial. El proyecto personal se reactiva en la sociedad occidental como un ejercicio de autonomía de cada sujeto, «algo diferenciado pero compatible con los proyectos colectivos» (Roca, 2006, p.131). Psicológicamente, el proyecto personal aplicado al deportista debe centrarle, saber qué desea y motivar su existencia. Debe estar razonablemente fundamentado y aceptando ritmos de logro distintos al resto de la población. Wylleman \& Lavallee(2004) exponen las transiciones del deportista con la edad: el desarrollo fisiológico, la dedicación al entrenamiento, las exigencias educativas y las relaciones 
psicosociales. El proyecto personal individualiza dichas transiciones vitales y acrecienta un desarrollo psicológico, social y académico totalmente particularizado (Balagué, 1999; Cruz, 1992). La adaptación académica del proyecto personal aplicado a los deportistas de alta dedicación se ha presentado recientemente con el nombre de Excelencia y Transiciones (Regüela, 2011). La amplitud del proyecto personal se refleja en Torregrosa et al. (2004) cuando destacan que el deportista de elite no debe ser valorado exclusivamente por sus rendimientos deportivos.

A nivel jurídico debe destacarse la normativa legal sobre educación y deporte. El Decreto 143/2007, de 26 de junio, para la ordenación de la educación secundaria obligatoria, refiere las medidas para facilitar el seguimiento de los estudios al alumnado con una dedicación significativa al deporte. Por otro lado, el Decreto 337/2002, de 3 de diciembre, sobre el alto rendimiento deportivo, establece el seguimiento académico en la tecnificación deportiva. En el alto rendimiento, se expone la atención académica (estudios, tutoría y seguimiento) en la enseñanza superior, con el acceso a plazas reservadas en las enseñanzas universitarias y no universitarias. Los deportistas están exentos de pruebas de acceso, en iguales condiciones que los deportistas de alto nivel del Consejo Superior de Deportes (CSD), según el Real Decreto 971/2007, de 13 de julio, sobre deportistas de alto nivel y alto rendimiento. Por este motivo, el artículo se titula deporte de alta dedicación, término que integra los deportistas con reconocimiento oficial y a los que, entrenando horas semejantes, carecen de dicho reconocimiento.

Lanormativa vigente incide también en los modelos de compaginación existentes. El modelo no integrado mantiene separadas las relaciones sociales de la escuela y del deporte, como dos ámbitos inconexos. Implica un gran esfuerzo personal y familiar para mantener ambas actividades en alto rendimiento, siendo además el modelo más generalizado. Las ayudas institucionales del modelo no integrado quedan reservadas a los alumnos con reconocimiento deportivo oficial que, en función de su nivel, podrán reconocer asignaturas o reservar plazas en las etapas educativas. Sin embargo, el artículo focaliza el estudio de las medidas de compaginación en forma de programas curriculares especí ficos o que sintetizan la escuela y el deporte en un mismo espacio. Con estas características encontramos dos tipos de modelos, ambos con referencias jurídicas (Solà, 2010):

a. Modelo no integrado con proyecto para deportistas, que mantiene las características del modelo no integrado, pero la escuela desarrolla un programa curricular adaptado y con el apoyo de la administración educativa. En el sistema público destacan los centros de especial atención a la práctica deportiva, aunque también existen centros privados con proyectos para deportistas.

b. Modelo integrado, que se basa en la localización en un mismo centro de la actividad académica y deportiva, alejando al estudiante de su entorno de proximidad, homogeneizando sus vivencias sociales. En el sistema público destacan los centros de alto rendimiento; a nivel privado, existen algunos clubs de alto nivel deportivo que integran el sistema educativo en su funcionamiento. El modelo integrado tiene referencias claras también en las medidas académicas específicas para música y danza (centros de enseñanza integrada).

El objetivo del artículo es comprender la compaginación de estudios y deporte en secundaria en el modelo educativo con proyecto y en el modelo integrado, evaluando también la implicación de los clubs. El estudio se basa en ocho indicadores extraídos de las entrevistas a los directores de los centros educativos (origen, titularidad, acceso de los estudiantes, calidad educativa, exención de la educación física, tutor personalizado, calendario y oferta), junto con otros ocho indicadores extraídos de las entrevistas a los directores de los club deportivos (seguimiento y exigencia académica, dedicación absoluta 16 años, exámenes y entrenamientos, formación de técnicos, currículum adaptado, tiempo invertido en los desplazamientos y personalidad del deportista). El análisis inductivo del contenido se organizó en cuatro constructos hermenéuticos: modelos educativos, proyectos de centro para deportistas, paralelismos con los estudios de música y danza, y la flexibilidad horaria y de calendarios. La auténtica implicación social del estudio es mejorar la efectividad de los modelos educativos para deportistas.

\section{Método}

Muestra

Participaron cuatro directores de los centros que ofrecen compatibilizar los estudios con los deportes tanto en la enseñanza pública como privada: dos representativos del modelo no integrado con proyecto y dos representativos del modelo integrado. Dos de ellos situados en Barcelona, uno en Sant Cugat del Vallés y otro en Sabadell. Se entrevistó también a la inspección educativa, que debe velar por la calidad de las propuestas $(n=5)$. La muestra se completa con entrevistas a cinco directores técnicos de un deporte de equipo (baloncesto) y a cuatro directores técnicos de clubs de un deporte individual (natación), con exigencias muy diferenciadas $(\mathrm{n}=9$ ). Todos los clubes representados son de alto nivel deportivo en cada especialidad y en las categorías de formación correspondientes con la educación secundaria. Todos los clubes con continuas presencias en los Campeonatos de España y con múltiples títulos o pódiums logrados. En total la muestra de entrevistas entre educación y deporte fue de 14 (Figura 1).

\begin{tabular}{|c|c|c|}
\hline Educación: Titularidad & $\begin{array}{l}\text { Modelo no integrado } \\
\text { con proyecto }\end{array}$ & Modelo integrado \\
\hline$N=5$ & \multicolumn{2}{|c|}{ Coinproyeco } \\
\hline $\begin{array}{l}\text { Pública } \\
\text { Privada } \\
\end{array}$ & $\begin{array}{l}\text { IES Ferran Tallada } \\
\text { STUCOM } \\
\end{array}$ & $\begin{array}{l}\text { IES Centro Alto Rendi miento } \\
\text { Escola Santa Clara }\end{array}$ \\
\hline Deporte: Clubs & Natación & Baloncesto \\
\hline \multirow{4}{*}{$N=9$} & Club Nat ació Sant Andreu & F.C. Barcelona Bàsquet \\
\hline & Club Esportiu Mediterrani & Club Joventut Badalona DKV \\
\hline & Club Natació Manresa & CB Unió Man resana \\
\hline & Club Nat ació Atlètic Barceloneta & Unió Esportiva Mataró \\
\hline $\mathrm{N}=\mathbf{1 4}$ & & CB Femení Sant Adrià \\
\hline
\end{tabular}

\section{Instrumentos}

Desde un enfoque cualitativo y descriptivo, se extrajeron datos mediante entrevistas con el fin de comprender las propuestas con ayudas institucionales para estudiantes deportistas y el funcionamiento de los clubs al respecto (Hernández, Fernández \& Baptista, 2004). Atendiendo a las apreciaciones de directores educativos, directores técnicos deportivos y estudiantes deportistas con la etapa secundaria finalizada (comité de expertos), se construyeron los guiones de dos entrevistas semi-estructuradas para recoger las opiniones de los profesionales. Ambas entrevistas constaban de ocho preguntas agrupadas en tres bloques: dos sobre la génesis/estructura; cuatro sobre las medidas concretas de compaginación estudios y deporte y, finalmente, dos sobre prospectiva de futuro.

\section{Procedimiento}

Se contactó telefónicamente con los directores de los centros escolares de referencia, con la inspección educativa, y con los directores técnicos de los clubs escogidos, explicándoles el objetivo del estudio y el interés de su participación. El día de la entrevista, se entregaba la carta de las fundaciones patrocinadoras y se hacía lectura previa de las preguntas. Las entrevistas fueron realizadas por el mismo investigador, con experiencia previa en la metodología. Los mínimos éticos exigibles se garantizaban con el anonimato de los datos obtenidos y el compromiso de no publicarlos sin consentimiento autorizado. Las entrevistas fueron grabadas en archivos de sonido digitales. La duración media de la entrevista era de aproximadamente una hora, permitiendo al interlocutor la máxima expresión en sus respuestas y una recopilación exhaustiva de datos. Los archivos de sonido digital se transcribieron en Verbatim, y utilizando el hardware informático SoundScriber que ralentiza el ritmo sonoro, se trasladó a texto mediante el procesador Microsoft Word. Una vez transcritas las entrevistas, antes de ser utilizadas en el estudio, se reenviaron a los correspondientes interlocutores para que repasaran sus respuestas o para atender algún error en la transcripción.

Con los datos narrativos de las entrevistas estructurados en 16 indicadores, se indujeron cuatro categorías de análisis hermenéutico mediante la utilización del programa Atlas/ti (modelos teóricos de análisis). 


\section{Resultados}

Los resultados se presentan a partir de las tres categorías de la entrevista, escogiendo las respuestas más relevantes ejemplificadas mediante citas textuales, agrupando la información en indicadores cualitativos. Las opiniones seleccionadas son una realidad fragmentada de la entrevista; la comprensión holística de la misma exige la lecturaíntegra del texto o escuchar los archivos de sonido (material disponible por el autor).

\section{Educación: Entrevista directores centros docentes con proyecto} deportistas

\section{Génesis y Evolución del Proyecto}

a. Origen

«Los colegios acaban diciendo: qué quieres hacer, ¿estudiar o hacer deporte? Apartir de aquí se genera un problema familiar, de entrenador, con la escuela».

«Hemos cambiado una vocal: no es entreno o estudio, es entreno y estudio... Cambiar esta vocal implica construir un proyecto».

«Un nadador no catalogado de alto rendimiento, pero que entrene cada día, tiene las mismas exigencias que aquel nadador que ya ha hecho lamarca».

Los modelos integrados son institucionales; los otros modelos nacen de inquietudes personales. Excepto el CAR, todos los proyectos nacen ligados a un deporte (natación, tenis, judo), para diversificarse posteriormente. Los privados compaginan una sola etapa educativa (Educación Secundaria Obligatoria ESO o Bachillerato), pero los públicos ofertan todas las etapas en sus Institutos de Educación Secundaria (IES).

\section{b. Titularidad}

«Se tiene que educar al deportista para que haga compatible el deporte con los estudios; no lo tenemos que educar para pedir compatibilidad. Tienen razón cuando se quejan que ambas actividades no son compatibles: es verdad, no son compatibles».

«[modelo integrado]...sólo es un modelo bueno por este mínimo sector de la población deportiva, pero nunca es un modelo universalmente bueno».

«Estos convenios se hacen con buena voluntad y con poco carácter institucional: quién tiene derecho a firmarlos, como se hacen... y, sobre todo se han divulgado poco.»

La evolución del proyecto depende del contexto del centro (titularidad y gestión), y de las relaciones externas para divulgarlo (familias interesadas, clubes, federaciones). La poca definición legal facilita la autonomía de cada centro para hacer progresar su modelo en número de deportistas atendidos, en una alta valoración del mismo.

\section{Medidas de Compaginación}

a. Acceso de los estudiantes

«Tienen que estar federados y hacer un mínimo de entrenamientos semanales de 9 horas de lunes a viernes...con el beneplácito del Departamento y de la Inspección».

«Sería muy restrictivo hasta los 12 años y medianamente restrictivo en $1 \mathrm{r}$ ciclo ESO (12-14 años); permitiría claramente compatibilizar en $2^{\circ}$ ciclo ESO (14-16 años)».

El acceso a los proyectos no integrados es menos restrictivo, ofertándose a todo el segmento de población que dedica muchas horas al entrenamiento deportivo. Los proyectos integrados seleccionan deportistas con mejor marca y posible mejor futuro.

\section{b. Exención de la Educación Física (EF)}

«... obviar la nota de EF es un prejuicio, se quita una opción a subir nota».

«La EF no es sólo «física», es educación social, espiritual y en valores, trabajo en equipo, cultura del esfuerzo... no tendría que ser una convalidación automática».
La exención de la EF empeora las notas de los estudiantes deportistas y pierden educación en valores. La realidad muestra estrategias no suficientemente reguladas para ahorrarse la asignatura. Además, es difícil comparar el deporte no académico con las claras exenciones de la música y la danza. Los modelos integrados exigen exención, puesto que su práctica puede ser contraproducente con los entrenamientos.

c. Tutor de deportistas

«...el subdirector tiene reducción de dos horas para toda la escuela; el tutor deportivo tiene 5 horas semanales para sólo estos 10 alumnos de élite: está todo dicho».

«...cada dos deportistas existe un tutor... Tiene el mismo sueldo, menos alumnos, horarios diferentes y muchas veces una sensibilidad especial hacia el deporte».

«No existe. El Jefe de Departamento, profesora de EF, emplea esas horas a la tutoría... a nivel interno, es la coordinadora del Proyecto de Deportistas».

«...existe el cargo no reconocido por el Departamento, implica muchas horas de trabajo y la escuela con sus propios recursos no puede retribuirlo debidamente».

El tutor de deportistas es la pieza clave de un buen proyecto, mediador que equilibra intereses de estudio y deporte. Se reconoce que el número de horas son insuficientes y que la remuneración debería mejorar, pero los proyectos no cuentan con dotación. Se cubre con cargos reconocidos (jefe de estudios o de departamento...), ya que no se reconoce esta figura ni siquiera en institutos con proyecto para deportistas.

\section{d. Calidad educativa}

«...seleccionar un nadador es seleccionar un rendimiento y una actitud «.

«Si el programa tiene éxito a largo plazo es porque el resultado académico de los alumnos deportistas será muy bueno. Si suspenden Selectividad es un fracaso absoluto».

«...El profesor de química pone notas a los deportistas y a los que no lo son».

«...la exigencia académica para la obtención del título tiene que ser igual. Después ya se encargará el mercado de decir si uno es mejor que el otro».

Nadie admite inferior exigencia académica al deportista, sería un engaño y una renuncia. La escuela presenta filtros para superar las etapas educativas, especialmente en el acceso a la universidad. Ninguna familia debe aceptar una mala preparación académica para desarrollar la carrera deportiva de un hijo.

\section{Prospectiva}

a. Calendario

«...lógico sería hacer calendarios escolares según las exigencias deportivas...».

«Deporte y educación son dos mundos independientes, no se hablan. El esfuerzo sólo lo hace la educación y sólo en algunos centros muy concretos».

«...se puede alargar el recorrido académico, pero no la vida única deportiva. La competición y el rendimiento están claramente establecidos: el deporte no es flexible.»

«Te diré más: ¡hay Comunidades Autónomas (CC.AA.) que la Consejería de Educación es la misma Consejería de Deportes, y también pasa esto!»

Todo el esfuerzo de compaginación recae en la educación, más maleable y dialogante. El deporte programa entrenamientos en función de calendarios de competición independientes de los ritmos educativos, incluidos periodos de examen.

b. Oferta y marketing

«Mirárselo en términos de competitividad es equivocado. Al contrario, se debe hacer grande el deporte y así será más grande el Club...»

«... te diré que ojalá existieran muchas escuelas que tuvieran esta 
sensibilidad».

«...hay lugar para los que estamos y 10 veces más: la oferta es corta y las familias hacen lo que pueden. Chicos de 10-16 años entrenando 10-15 h. hay muchos «.

«...tener más centros con proyecto de deportistas estaría muy bien, significaría que hay muchos jóvenes practicando deporte de nivel y, además, estarían controlados».

Los entrevistados no temen una futura competencia entre centros docentes. La idea es juntar esfuerzos porque hay espacio para nuevas propuestas. Aún hay muchos estudiantes deportistas sin la atención que requiere su esfuerzo cotidiano. El análisis del contenido de las entrevistas permite una compilación inductiva de la información que contraste ambos modelos educativos (Figura 2):

\begin{tabular}{|c|c|c|}
\hline \multicolumn{3}{|c|}{ Diferencias y similitudes entre modelos educativos } \\
\hline $\begin{array}{l}\text { Diferencias y similitudes } \\
\text { en los indicadores }\end{array}$ & $\begin{array}{c}\text { Modelo no integrado } \\
\text { con proyecto }\end{array}$ & $\begin{array}{l}\text { Modelo } \\
\text { Integrado }\end{array}$ \\
\hline \multicolumn{3}{|c|}{ Diferencias } \\
\hline a. Origen & $\begin{array}{l}\text { Origen personal vinculado a un } \\
\text { único deporte }\end{array}$ & $\begin{array}{l}\text { Origen institucional y } \\
\text { multideporte }\end{array}$ \\
\hline b. Titularidad & \multicolumn{2}{|c|}{$\begin{array}{l}\text { Pública: disponen de tod as las etap as educativas y regímenes de } \\
\text { formación. } \\
\text { Privada: limitados a una única etapa educativa, sin posibilidad de } \\
\text { continuidad. }\end{array}$} \\
\hline c. Acceso estudiantes & Condiciones mínimas de acceso & Condiciones máximas de acceso \\
\hline d. Exención Ed. Física & $\begin{array}{l}\text { En contra, va en det rimento de la } \\
\text { nota media }\end{array}$ & $\begin{array}{l}\text { A favor, incompatibilidad con } \\
\text { entrenamientos }\end{array}$ \\
\hline \multicolumn{3}{|c|}{ Simi litudes } \\
\hline e. Tutor personalizado & \multicolumn{2}{|c|}{$\begin{array}{l}\text { Imprescindibles para los proyectos, pero es casos recurs os y falta de } \\
\text { reconocimiento. }\end{array}$} \\
\hline f. Calidad educativa & \multicolumn{2}{|c|}{ Ningún centro docente acepta la rebaja de la exigencia académica. } \\
\hline g. Calendario & \multicolumn{2}{|c|}{$\begin{array}{l}\text { La flexibil idad educativa no si empre puede pal iar el inmovilismo del } \\
\text { depo ite. }\end{array}$} \\
\hline h. Oferta y marketing & \multicolumn{2}{|c|}{$\begin{array}{l}\text { Si exis te competen cia de oferta en el futuro significará la evo lución de } \\
\text { la compatibilización. }\end{array}$} \\
\hline
\end{tabular}

Deporte: Entrevista directores técnicos de clubs de alto rendimiento

Estructura de Cluby Seguimiento Académico

a. Seguimiento académico

«El club repasa cada trimestre las notas obtenidas por sus jugadores en las diferentes evaluaciones. Lo tienen que hacer los mismos entrenadores de los equipos».

«Desde la Escuela hasta la categoría Júnior, el club revisa las notas trimestrales. Si no son buenas, se penaliza al jugador sin entrenar un día, o sea, jugar menos en el partido».

«No hay una política específica de club que controle el seguimiento académico de los alumnos; en principio es responsabilidad de los padres».

«El club no realiza control de notas a los nadadores, esto se deja a cada familia».

Sólo dos potentes clubs de baloncesto se implican en el control trimestral de las notas de sus jugadores, aportando medidas concretas para incentivar el estudio. Contrariamente, en natación hay respuesta homogénea: los clubs no se hacen cargo del control académico de los nadadores ya que se considera una obligación de las familias.

\section{b. Exigencia académica}

«Si un jugador no saca buenas notas se le «castiga» sin jugar; también lo tendrían que hacer las respectivas selecciones, por qué si no siempre es el club quien carga con la responsabilidad académica...»

«En baloncesto, la pérdida de practicantes en edades avanzadas no es un problema académico, es un problema de selección de los jugadores».

«...los motivos para dejar la natación son dos: primero, el progresivo incremento de la exigencia en los estudios y, como consecuencia, la bajada en las sesiones de entrenamiento, y el rendimiento en el agua disminuye».

«La combinación dela exigencia académica junto con la dificultad de mejorar el rendimiento cuando pasan de categoría provoca pérdidas de motivación hasta dejarlo»
La pregunta es más pertinente en la natación donde la respuesta es unánime entre los cuatro directores técnicos: el progresivo aumento de la exigencia escolar no permite seguir con el ritmo de entrenamientos que asegure el buen rendimiento deportivo.

\section{Medidas de compaginación}

a. Dedicación absoluta al deporte a los 16 años

«Si deja de estudiar sólo deslumbrado por un futuro deportivo, no se aceptaría. Estos problemas surgen cuando un jugador está en manos del representante: se vuelve egoísta y no piensa en el equipo»

«Los jugadores tienen que saber que sólo alguno de ellos llegaráa ser profesional de baloncesto y, por lo tanto, tienen que estudiar».

«No es opinable; el club afirma la obligación de asistir a la escuela».

«Tienen que continuar estudiando: No se beca nunca ningún jugador sin la exigencia del estudio».

«Desgraciadamente en la natación esto pasa, pero el club no lo aconsejaría. Ni siendo nadador de nivel europeo dejaría los estudios. Quizás se podría hablar a nivel mundial u olímpico...».

«El club entiende que los estudios no se pueden dejar, pero acaban decidiendo los mismos nadadores y las familias».

«Depende de su nivel. Si su nivel no es demasiado alto, le recomendaría que no lo dejara...Si es de nivel, formarse sin que le afectara al programa de entrenamiento».

«No se permitiría. Los nadadores han de entender que deben estudiar un oficio o una carrera, no se ganarán la vida con la natación aunque sean muy buenos».

Los comentarios de los directores técnicos en baloncesto son muy elocuentes y claros. Hay total consenso respecto de no dejar nunca los estudios. La cuestión presenta mucha más permeabilidad en la natación. No se niega drásticamente la posibilidad de dejar los estudios a los 16 años en función del rendimiento y las marcas del deportista. ¿Se asume la imposibilidad, a una cierta edad, de poder compaginar estudios y deporte?

\section{b. Examen y entrenamiento (competición)}

«...no hay problema en perder un entrenamiento por examen; se puede compensar durante la semana haciendo otra sesión con la categoría superion».

«Se propuso entrenar en sábado por la mañana para recuperar entrenamientos perdidos en la semana por estudios pero...las concentraciones del domingo con las selecciones hacen que el jugador no tenga tiempo ni en el fin de semana»

«Evidentemente que si un día hay un examen importante, no pasa nada por no venir al entrenamiento; además, podemos adaptarle aquel entrenamiento a otro día de la semana. De todas maneras, el nadador debe aprender a gestionar su tiempo, un tiempo diferente del joven que no hace deporte de competición».

«Si está preparando una competición muy importante, le recomendaría entrenar. Si es un nadador de nivel medio, le obligaría a preparar el examen...»

Se muestra un alto grado de flexibilidad si hay que dejar puntualmente un entrenamiento para estudiar o por un examen. De todas maneras, se relativiza esta idea si el examen coincide con campeonatos muy importantes (hecho habitual desgraciadamente en natación), momento que la falta al entrenamiento pueda afectar.

\section{c. Formación de técnicos}

«...sobre todo en los jugadores que empiezan, coincidir con un entrenador preparado que al margen del baloncesto también sepa educarlos, mucho mejor».

«Se exigen los títulos de entrenadores correspondientes, como mínimo. Al margen de la formación específica, otros son estudiantes universitarios de MEF y otras licenciaturas, hecho que también se valora por aportar ciertos valores a los jugadores».

«Es muy importante tener entrenadores con experiencia y estudios. Todos los entrenadores tienen titulación deportiva y la mayoría son licenciados universitarios». 
«En primer lugar, se mira la validez de la persona independientemente de su título. Es bueno que los entrenadores jóvenes se enriquezcan con gente más experta...»

Criterios: disponer de titulaciones deportivas como condición prácticamente indispensable; al margen de los estudios, valorar el trato personal del entrenador (importancia de tener un entrenador sensible e implicado en la iniciación), y, finalmente, no parece relevante la formación académica o universitaria.

\section{d. Currículos adaptados}

«...presioné mucho para que el deporte apareciera en la ley, igual que la música y la danza; ha aparecido algo, pero del todo insuficiente y poco concreto"...

«El problema es que una escuela de danza o un programa de música, establecen cursos con una determinada carga lectiva... En el deporte, el número de horas a entrenar no está definido tan claramente y no tiene rango académico...»

«Danza y Música están muy bien compatibilizadas, pero la variabilidad de deportes hace difícil establecer una ley general... La mejor solución actual es justificar muy bien cada caso particular, a título personal, y buscar apoyo institucional».

Respuestas unánimes: imposibilidad de comparar el deporte, considerado no académico, con las claras exenciones de la música o la danza. Se aprecian múltiples «prácticas oscuras» no reguladas para ahorrarse la asignatura educación física.

\section{Prospectiva}

a. Tiempo de desplazamientos

«Hay jugadores de diferentes ciudades: Reus, Girona, Manresa, Sabadell... Evidentemente, estos jugadores todavía tienen menos tiempo para estudiar, puesto que tienen que invertirlo en los desplazamientos».

«Hay varios jugadores que vienen de fuera... Para que no pierdan el tiempo, el club dispone de un aula de estudio para hacer deberes antes o después de entrenar. También se consideró el hecho de tener un profesor de apoyo, pero la idea no ha encajado (diferentes categorías, edades, movimientos de jugadores...)».

«Tenemos bastantes nadadores de fuera del barrio y también de fuera de la capital. Gente que hace esfuerzos muy grandes para poder venir a entrenarse».

Los clubes de baloncesto tienen jugadores de fuera de su ciudad, con distancias de desplazamiento considerables (50-125 Km). Sólo un club ha mostrado posibles soluciones académicas para cubrir las horas que se pasan en el club. En natación no hay tantos nadadores de fuera y, de tenerlos, las distancias son más cortas.

b. Trabajar la personalidad del deportista.

«...a menudo, los que son mejores en el agua también lo son en los estudios».

«...se confirma que si un jugador es mal estudiante no depende del

\begin{tabular}{|c|c|c|}
\hline \multicolumn{3}{|c|}{ Diferencias y simil itudes entre dubs deportivos } \\
\hline $\begin{array}{l}\text { Diferencias y similitudes } \\
\text { en los indicadores }\end{array}$ & $\begin{array}{l}\text { Deporte de Equipo: } \\
\text { Clubs de baloncesto }\end{array}$ & $\begin{array}{l}\text { Deporte Individual: } \\
\text { Clu bs de natación }\end{array}$ \\
\hline \multicolumn{3}{|c|}{ Diferencias } \\
\hline a. Segui miento académico & Control de notas & Responsabilidad de las familias \\
\hline b. Exigencia académica & No afecta la retirada prematura & Afecta a la retirada premat ura \\
\hline c. Dedicación absoluta 16 años & No es aceptab le en ningún caso & $\begin{array}{l}\text { Es posible aceptarlo en ciert os } \\
\text { casos }\end{array}$ \\
\hline g. Tiempo de desplazamientos & $\begin{array}{l}\text { Significativos y con ayudas del } \\
\text { club }\end{array}$ & $\begin{array}{l}\text { Cortos. Ayudas sólo en la máxima } \\
\text { categoría }\end{array}$ \\
\hline \multicolumn{3}{|c|}{ Similitudes } \\
\hline $\begin{array}{l}\text { d. Examen y entrenamiento } \\
\text { (competición) }\end{array}$ & \multicolumn{2}{|c|}{$\begin{array}{l}\text { Se puede faltar al entrenamiento, pero siem pre que sea posible se } \\
\text { recuperará }\end{array}$} \\
\hline e. Formación de técnicos & \multicolumn{2}{|c|}{ Importante la formación deportiva espećífica, universi taria y moral } \\
\hline f. Curículos adapt ados & \multicolumn{2}{|c|}{ Dificul tades de compaginación sin modelos de actuación claros } \\
\hline h. Pers onali dad del deporti sta & \multicolumn{2}{|c|}{ No existe un deportista y un es tudiante: son la misma pers ona } \\
\hline
\end{tabular}

deporte».

«...se constata que los que no valen para los estudios no les hace falta ninguna ayuda extra, puesto que muchos de ellos siguen suspendiendo».

«Si eres disperso en la vida, eres disperso en el baloncesto».

En el deportista no existen dos personas diferentes, una académica y otra competitiva. Siempre es la misma persona, y sus valores, esfuerzos y motivaciones se trasladan de la escuela al deporte. En principio, el deporte no se constata como causa de un posible descenso del rendimiento escolar.

\section{Discusión}

Los resultados ponen de manifiesto que, aunque en los últimos años ha habido concienciación de las dificultades de los jóvenes deportistas para compaginar entrenamientos y estudios, los modelos educativos están aún en fase de desarrollo, mostrando problemas comunes de apoyo legal o de flexibilización de las medidas pedagógicas aplicables. Se profundiza en la comprensión de la compaginación entre centros docentes y clubs deportivos, configurando la discusión en cuatro categorías de análisis hermenéutico vinculadas a los 16 indicadores de las entrevistas (Figura 4):

\begin{tabular}{|c|c|c|}
\hline $\begin{array}{c}\text { Indicado res } \\
\text { Centros Docentes }\end{array}$ & Categoría & $\begin{array}{c}\text { Indicadores } \\
\text { Clubs Deportivos }\end{array}$ \\
\hline Génesis & \multirow{3}{*}{ MODELOS EDUC ATIVOS } & Estructura \\
\hline Origen & & Seguimiento académ ico \\
\hline Titularidad & & Exigencia académ ica \\
\hline Medidas de compaginación & & Medidas de compaginación \\
\hline Acceso estudi antes & \multirow{2}{*}{ PROYECTOS DE CENTRO } & Dedicación absoluta 16 \\
\hline Calidad educativa & & Examen y entrenamiento \\
\hline Tutor personalizado & \multirow{2}{*}{ ESTUDIOS DE MÚSICA Y DANZA } & Formación de técnicos \\
\hline Exención Ed. Física & & Currículos adaptados \\
\hline \multicolumn{2}{|l|}{ Prospectiva y marketing } & Pros pectiva \\
\hline Calendario & & Tiempo des plazamientos \\
\hline & $\begin{array}{l}\text { ENTRENAMIENTOS, } \\
\text { DESPLAZAMIENTOS, }\end{array}$ & \\
\hline Oferta y market ing & COMPETICIONES & Personalidad deportista \\
\hline
\end{tabular}

Figura 4. Model os teóricos de análisis.

\section{Modelos Educativos}

Las resoluciones relativas a la organización de los centros públicos de secundaria, establecen dos posibilidades de compaginación para la ESO y el Bachillerato. La primera para los alumnos que ya estudian en un centro con proyecto (especial atención a la práctica deportiva); la segunda, dirigida a los alumnos que no pertenecen a la primera tipología de centros, y que sólo tienen derecho a flexibilización curricular en virtud de su pertenencia al programa ARC (Alto Rendimiento de Cataluña). El resto de alumnos deportistas quedan excluidos (Figura 5):

Ambas modalidades educativas ofrecen exiguas posibilidades a la mayoría de estudiantes deportistas. Excepcionalmente las resoluciones de 15 de junio de 2010 y la vigente de 21 de junio de 2013, incluyen que en casos excepcionales de dedicación intensiva al deporte, aunque el alumno no tenga el certificado ARC, pueda optar a un currículum individualizado. Importante párrafo que abre la posibilidad de compaginar a pesar de no pertenecer a dicho programa, aunque la imprecisión normativa es evidente. Ante las escasas posibilidades de acceder a un modelo educativo para deportistas, se destaca que los clubs puedan realizar también un seguimiento pedagógico del rendimiento escolar para compensar la falta de ayudas generalizadas. El deporte no puede mantenerse ajeno en su implicación en la escuela en una compaginación que apunte a la excelencia.

\section{Proyectos de Centropara Deportistas}

Si el desarrollo de un proyecto permitiera a un centro educativo su integración en la red de especial atención a la práctica deportiva, se democratizaría el modelo, acercando los institutos a los deportistas 


\begin{tabular}{|c|c|c|}
\hline $\begin{array}{l}\text { Centros docentes de educación } \\
\text { secundaria }\end{array}$ & $\begin{array}{l}\text { ESO } \\
\text { Dedicación significativa a la } \\
\text { práctica del deporte }\end{array}$ & $\begin{array}{l}\text { Bachillerato } \\
\text { Compaginar con la práctica } \\
\text { intensiva del dep orte }\end{array}$ \\
\hline $\begin{array}{l}\text { Centros de especial atención a la } \\
\text { práctica deportiva }\end{array}$ & \multicolumn{2}{|c|}{ Proyectos de centro con adapt aciones del curń culu m } \\
\hline $\begin{array}{c}\text { Otros centros } \\
\text { Docentes }\end{array}$ & \multicolumn{2}{|c|}{ Pertenecer al Programa de Tecnificación ARC } \\
\hline \multicolumn{3}{|c|}{ Figura 5. Etapas educativas y centros para compaginar estudios y deporte. } \\
\hline $\begin{array}{l}\text { Es tudios de música } \\
\text { y danza }\end{array}$ & Factores de comparación & Deportistas de competición \\
\hline $\begin{array}{c}\text { Sí } \\
\text { (ESO y Bachillerato) }\end{array}$ & $\begin{array}{l}\text { Reducción por convalidación o } \\
\text { reconocimiento }\end{array}$ & $\begin{array}{c}\text { No } \\
\text { (muy limitado) }\end{array}$ \\
\hline 3-15h semanales & Horas de dedicación & $5,81 \mathrm{~h}-16,14 \mathrm{~h}$ \\
\hline Prueba de acceso & Criterios de admisión ayudas & Acreditar marcas \\
\hline Fommativo & Criterio & Resul tadi sta \\
\hline $\begin{array}{l}\text { Conservat orio, centro profesional } \\
\text { o escuela }\end{array}$ & Tipos de centro & $\begin{array}{l}\text { Clubs deportivos, } \\
\text { Centro de tecrificación }\end{array}$ \\
\hline $\begin{array}{l}\text { Enseñanzas oficiales en régimen } \\
\text { especial }\end{array}$ & Enseñanzas & No enseñ anzas oficiales \\
\hline $\begin{array}{l}\text { Modelo integrado y } \\
\text { no integrado }\end{array}$ & Modelo educativo & $\begin{array}{l}\text { Mo delo integrado y no } \\
\text { integrado con proyecto }\end{array}$ \\
\hline
\end{tabular}

(Vilanova, 2009). Si institucionalmente no se dan a conocer los proyectos, el éxito de compaginar estudios y deporte en la escuela pública y privada es escaso. El modelo integrado reduce el dobleámbito social de la escuela y del club al localizar en un mismo centro ambas actividades (Cano, 2008; Solà, 2010). La presencia de institutos en los centros de alto rendimiento es una solución válida para aquellos pocos deportistas que disfruten del modelo integrado (Flanagan \& Winther, 2004).

$\mathrm{Al}$ margen de las condiciones de acceso expuestas, la opción de encontrar una escuela con proyecto deportivo tampoco garantiza permanecer en el contexto habitual. Las familias satisfechas con la escuela elegida, demoran este paso hasta que ambas exigencias se hacen insostenibles y se plantea el cambio de proyecto personal del joven deportista (Roca, 2006; Regüela, 2011). La calidad académica de los proyectos no rebaja en absoluto el listón de la exigencia cultural; no hay respuesta más unánime entre los entrevistados.

Los clubs presentan estrategias para compensar un entrenamiento perdido por exigencias de exámenes y no se conforman con la finalización de la escolarización obligatoria (16 años) para dar por concluida la formación del deportista y exigirle dedicación absoluta. Sin embargo, la unanimidad de estos criterios puede resquebrajarse según el nivel deportivo o ante una exigencia competitiva de máximo nivel.

\section{Estudios de Música y Danza}

Es recurrente la comparación con las detalladas modificaciones curriculares de los alumnos que cursan simultáneamente estudios de música o danza. La regulación de las exenciones del bachillerato para el alumnado con práctica intensiva del deporte carece de la misma precisión. La claridad legislativa en música y danza tendría que ser equitativa para los deportistas: «(...) analogía entre los estudios de danza o música y los centros educativos de especial atención a la práctica deportiva, en aquello que establece la normativa...» (Cano, 2008, p.174). Semejantes horas de dedicación que los deportistas desarrollan sin ayudas institucionales (Solà, 2010). El deportista debe tener marcas para obtener beca, criterio resultadista. En cambio, la admisión en música y danza se basa en una prueba con criterio formativo. Las diferencias surgen de comparar centros oficiales con clubs deportivos sin reconocimiento académico. La normativa para música y danza promueve un modelo no integrado con proyecto que coexiste con algunas propuestas integradas, especialmente en danza (Figura 6).

La exención de la Educación Física es la medida básica, pero se antoja demasiado simple: perjudica las notas y se pierden sus valores educativos. Además, los alumnos sin los requerimientos legales se acogen a acuerdos particulares sin oficialidad para conseguir horas de compaginación. Una actuación coherente entre el tutor del programa educativo y el técnico deportivo ejemplificaría la solución natural. Además, al entrenador no se le debe valorar exclusivamente por los resultados deportivos. Las familias agradecen la preocupación para compatibilizar con los estudios y lograr una estabilidad que también revertirá en el rendimiento deportivo.

\section{Entrenamientos, Desplazamientos y Competiciones}

Básicamente, la compaginación debe atender a los entrenamientos, a los desplazamientos y a las competiciones. Además las horas de entrenamiento aumentan con la edad, paralelamente al aumento de la complejidad académica. Los desplazamientos cotidianos de muchos jóvenes deportistas restan horas al estudio y añaden fatiga. Algunos ejemplos de ida y vuelta a Barcelona, de 4 a 6 veces por semana: Girona (103 Km, 1h15'), Manresa (65 Km, 55'), Reus (109 Km, 1h25')... Torregrosa et al. (2004) incidían en que los desplazamientos alejados de la residencia familiar afectan a que los adolescentes olímpicos no acaben los estudios.

Los proyectos deberían flexibilizarse ante los periodos de competición. Destaca por su relevancia el 2. ${ }^{\circ}$ Bachillerato, con calendarios deportivo y académico incompatibles. Los estudiantes pasan los meses de abril, mayo y junio inmersos en las fases finales de los campeonatos autonómicos y de España, e incluso en competiciones internacionales. Competición de máximo nivel que coincide con los exámenes finales de bachillerato y de acceso a la universidad. Además se acumula el desgaste fisiológico por aumento de la calidad del entrenamiento y la disminución de la tolerancia del entrenador en el momento de máximo rendimiento (Heinemann, 1998).

Las limitaciones del estudio que sugieren futuras investigaciones para su mejora se sintetizan en los siguientes puntos:

a) Conocer la opinión directa de los estudiantes deportistas, recoger sus pensamientos y sus vivencias en función de la etapa educativa y la exigencia deportiva. Si realmente se quiere profundizar sobre las dificultades de los modelos educativos de compaginación, es lógico pensar en dar la voz a los estudiantes deportistas.

b) Además, completar dicho estudio comparándolo con las posibilidades efectivas de compaginación de los estudiantes de música y danza, actividades formativas reconocidas por el sistema educativo. Las horas de dedicación a la música y a la danza, al margen de teneruna mejor regulación normativa, revertirán en un reconocimiento académico y curricular que la dedicación al deporte no puede ofrecer.

c) Ampliar el espectro de la muestra de centros educativos públicos y privados tanto del modelo no integrado con proyecto como del modelo integrado, de los cuales no existen aún demasiados ejemplos, aumentando el rango geográfico de centros. Además, compararlo con otros clubs de alto nivel representativos de diferentes modalidades deportivas individuales y colectivas (tenis, fútbol...).

d) El título del artículo en relación a la educación secundaria, sin diferenciar la etapa obligatoria(ESO) de la post-obligatoria (Bachillerato o Ciclos Formativos) puede resultar demasiado genérico. Aunque en el texto se explicitan algunas diferencias, sería conveniente precisar con detalle las medidas concretas de compaginación que se puedan asumir en cada etapa educativa: ESO, Bachillerato, Formación Profesional, etc.

\section{Conclusiones}

Las condiciones de ingreso en los proyectos no integrados son menos restrictivas que las condiciones de ingreso en los proyectos integrados, que seleccionan a los mejores deportistas o a quienes tienen un mejor futuro (elitista de rendimiento deportivo). Reservadas a una élite deportiva con rendimientos actuales notables, se olvidan del resto de la población deportista que entrena un volumen de horas semejante. El modelo integrado implica romper con la vida social y familiar cotidiana, y, sin embargo, no exime totalmente de severas dificultades para congeniar la vida académica con el rígido calendario deportivo de competiciones o de entrenamientos.

Nunca se pagará peaje académico o cultural para permitir que el deportista pueda entrenar. Compatibilizar, precisamente quiere decir no renunciar. Ningún centro docente quiere tener alumnos a cambio que estos salgan mal preparados. Ninguna familia debería aceptar una renuncia académica a cambio de la carrera deportiva de un hijo.

El esfuerzo para compaginar estudios y deporte recae sobre la educación. El deporte programa entrenamientos en función de calendarios de competición independientes del calendario académico. Las 
disfunciones son continuas cuando se solapan exigencias de competición con periodos de examen o con entrega de trabajos. Para atenuar dichas disfunciones, un buen proyecto debe pivotar sobre un docente líder en la difícil tarea de compaginar los estudios con el deporte. El tutor de deportistas es la pieza clave del proyecto: debe dedicarle tiempo, tener especial sensibilidad con estos alumnos y templar las negociaciones entre las partes. Todos los modelos reconocen que el número de horas que pueden dedicarle son insuficientes y que la remuneración debería mejorar

No se distinguen adaptaciones curriculares por modalidades deportivas; son modificaciones genéricas que deben aceptar los diferentes deportistas (baloncesto y natación en nuestro artículo). Contra la idea generalizada de promover exenciones de asignaturas, el criterio más extendido parece que lo considera un prejuicio para la media de notas. Incluso la asignatura de educación física se contempla más allá del simple desarrollo orgánico; implica toda una educación en valores que no se debe despreciar y que el alumnado deportista puede enriquecer. Sin embargo, fuera del marco legal, existen pactos entre clubs y centros docentes, amparados por las familias, para obtener una nota de la asignatura sin tener que cursarla.

Ninguno de los modelos entrevistados temen por una futura competencia entre los centros docentes que ofrezcan programas para deportistas. El planteamiento es de juntar esfuerzos para mejorar el deporte autonómico y estatal. La estructura de club es más heterogénea, en función de los requerimientos de cada modalidad y a su idiosincrasia menos sujetos a una normativa general como sucede con los centros docentes.

\section{Referencias}

Balagué, G. (1999). Understanding identity, value and meaning when working with elite athletes. The Sport Psychologist, 13, 89-98.

B.O.E. (2007). Real Decreto 971/2007, de 13 de julio, sobre deportistas de alto nivel y alto rendimiento. B.O.E. $\mathrm{n}^{\circ} 177,25-7-2007$.

Cano, M. (2008). Com educar avui pel demà? Proposta per un model integral de centre d'innovació educativa. (Memoria de Investigación, Generalitat de Catalunya: Departament d'Educació). Recuperado dehttp://www.xtec.cat/ mcano/avui_x_dema/index1.html

Ceciæ Erpiè, S., Wylleman, P. \& Zupancic, M. (2004). The effect of athletic and non-athletic factors on the sports career termination process. Psychology of Sport and Exercise, 5, 45-59.

Cruz, J. (1992). El asesoramiento y la intervención psicológica en deportistas olímpicos. Revista de Psicología del Deporte, 2, 41-46.

D.O.G.C. (2002). DECRETO 337/2002, de 3 de diciembre, sobre el alto rendimiento deportivo. D.O.G.C. $n^{\circ} 3787,23-12-2002$.

D.O.G.C. (2002). DECRETO 143/2007, de 26 de junio, de ordenación de las enseñanzas de educación secundaria obligatoria.D.O.GC. $n^{\circ}$ 4915, 29-6-2007.

Flanagan, J. \& Winther, P. (2004). Combining sports and education: Support for athletes in the EU member Status. Documento de Trabajo del Parlamento Europeo.

Generalitat de Catalunya. Departamento de Educación (2010). Resolución de 15 de junio de 2010 para la organizacióny el funcionamiento de los centros públicos de educación secundaria para el curso 2010-2011.

González, Mª.D. \& Bedoya, J. (2008). Después del deporte, ¿qué? Análisis psicológico de la retirada deportiva. Revista de Psicología del Deporte, 17 (1), 61-69.

Heinemann, K. (1998). Einführung in die Soziologie des Sports. Schorndorf: Hofmann.

Hernández, R., Fernández, C. \& Baptista, P. (2003). Metodología de la Investigación. ( $3^{\mathrm{a}}$ ed.). México, McGraw-Hill Interamericana.

Murphy, G. M., Petitpas, A. J. \& Brewer, B. W. (1996). Identity foreclosure, athletic identity and career in intercollegiate athletes. Sport Psychologist, 10, 239-246.

Regüela, S. (2011). Programa Excel.lència i Transicions (ÈXITS) (Tesi Doctoral). Departament de Psicologia Bàsica, Evolutiva i de
l'Educació, Facultat de Psicologia: UAB. Recuperado de http:// www.tdx.cat/browse?value $=$ Reg\%C3\%BCela + S\% C3\%A1ez\%2C+Susana\&type=author

Roca, J. (2006). Automotivación. (1 $1^{\mathrm{a}}$ ed.). Barcelona, Paidotribo.

Solà, J. (2010)Academic Rigor and Dedication to Competitive Sport in Young People 12-18 Years: Major Social Issues. Physical Culture and Sports Studies and Research, Vol. L, 100-109.

Solà,J.(2010).El fràgil equilibrientrel'exigència acadèmicai la dedicació a l'entrenament de l'esport de competició en joves de $12-18$. Recuperado dehttp://www.fundacioernestlluch.org/ca/publicacio/ el-fragil-equilibri-entre-l-exigencia-academica-i-la-dedicacio-a-lentrenament-de-l-esport-de-competicio-en-joves-de-12-18

Singh, A., Uijtdewilligen, L., Twisk, J.W.R., Van Mechelen, W. \& Chinapaw, M.J.M. (2012). Physical Activity and Performance at School. A Systematic Review of the Literature Including a Methodological Quality Assessment. Archives of Pediatrics \& Adolescent Medicine, 166(1), 49-55.

Stambulova, N. (2000). Athletes' crises: A developmental perspective. International Journal of Sport Psychology, 31, 584-601.

Surís, J.C. \& Parera, N. (2005). Don’t stop, don't stop: physical activity and adolescence. International Journal of Adolescent Medicine and Health, 17 (1), 67-78.

Taras, H. (2005). Physical Activity and Student Performance at School. Journal of School Health, 75 (6), 214-218.

Torregrosa, M., Sánchez, X. \& Cruz, J. (2004). El papel del psicólogo del deporte en el asesoramiento académico - vocacional del deportista de élite. Revista de Psicología del Deporte, 13 (2), 215-228.

Vilanova,A. (2009). El procés d'inserció laboral d'esportistes olímpics a Catalunya, (Tesi Doctoral). Departament de Teoria i Història de l'Educació, INEFC: Universitat de Barcelona. Recuperado de

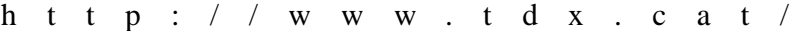
browse?value=Vilanova+Soler\%2C+Anna\&type=author

Vilanova,A., \& Puig, N. (2013). Compaginar la carrera deportiva con la carrera académica para la futura inserción laboral: ¿Una cuestión de estrategia?. Revista de Psicología del Deporte 22 (1), 61-68

Werthner, P. \& Orlick, T. (1986). Retirement experiences of sucessful olympic athletes. International Journal of Sport Psycology, 17, 337-363.

Williams-Ryce, B. T. (1996). After the final snap: cognitive appraisal, coping, and life satisfaction among former collegiate athletes. Academic Athletic Journal Spring, 30-39.

Wylleman, P., De Knop, P., Menkehorst, H., Theeboom, M. \& Annerel, J. (1993). Career termination and social integration among elite athletes. Comunicación presentada al 8th world congress of sport psychology, 902-905, Lisbon.

Wylleman, P. \& Lavallee, D. (2004). A developmental perspective on transitions faced by athletes. A M. Weiss (Ed.), Developmental sport and exercice psychology: A lifespan perspective (507-527). Morgantown, WV: Fitness Information Technology.

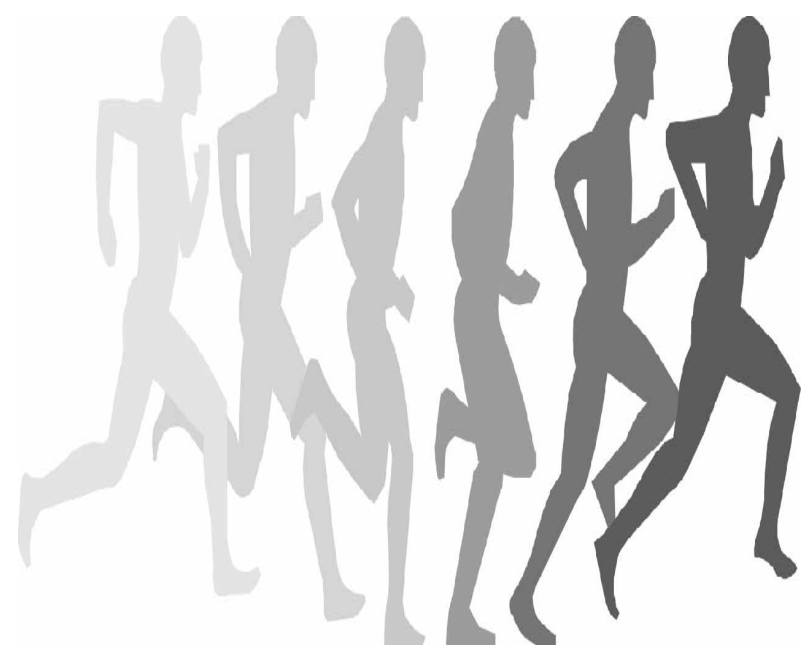

